ZU-TH- 16/1993

June 1993

\title{
Constraints on the Higgs and Top Quark Masses From Effective Potential and Non-Commutative Geometry
}

\author{
A. H. Chamseddine ${ }^{1,2} *$ and J. Fröhlich ${ }^{3} \dagger$ \\ ${ }^{1}$ Theoretische Physik, Universität Zürich, CH 8001 Zürich Switzerland \\ ${ }^{2}$ Université Aix-Marseille II, Luminy, France \\ ${ }^{3}$ Institut des Hautes Etudes Scientifiques, F-91440 Bures-sur-Yvette, France
}

\begin{abstract}
We consider the standard model in the formulationo of non-commutative geometry, for a Euclidean space-time consisting of two copies. The electroweak scale is set by the vacuum expectation value of a scalar field and is undetermined at the classical level. By adding the Coleman-Weinberg effective potential, that scale turns out to be fixed. Provided that the renormalized form of the Lagrangian maintains the vanishing of the cosmological constant, we show that the only solutions for the minimization equations of the total potential occur in the narrow band $146.2 \leq m_{t} \leq 147.4 \mathrm{Gev}$ for the top quark mass, with the corresponding Higgs mass $117.3 \leq m_{H} \leq 142.6$ Gev.
\end{abstract}

* Supported in part by the Swiss National Foundation (SNF)

$\dagger$ Permanent address: Theoretische Physik, ETH, CH 8093 Zürich Switzerland 
Connes' [1] framework of non-commutative geometry provides a geometrical interpretation of the Higgs field necessary to break the gauge symmetry spontaneously. The structure of space-time is taken to be a product of a continuous four-dimensional Riemannian manifold times a discrete set of two points. For such a structure of spacetime the usual methods of differential geometry fail and must be replaced with the more general framework of non-commutative geometry. Using the non-commutative setting, Connes and Lott [2] recovered the standard model with all its parameters, at the classical level. At present, it is not known how to quantize the non-commutative action directly. We are left only with the possibility of quantizing the resulting theory in the usual way. The quantum corrections are then given by familiar expressions. There is, however, one important difference between this approach and the standard analysis connected with the gravitational effects of the discrete geometry. This have, under certain conditions, very surprising consequences. Explaining and exploiting these effects is the main concern of this note. Geometrically, the distance between the two copies of the four-dimensional Minkowski space is the inverse of the electoweak scale. At smaller scales the flat manifold is curved and the distance between the two copies becomes a dynamical scalar field [3]. The leptonic Dirac operator associated with Connes-Lott space takes the form

$$
D_{l}=\left(\begin{array}{cc}
\gamma^{a} e_{a}^{\mu}\left(\partial_{\mu}+\ldots\right) \otimes 1_{2} \otimes 1_{3} & \gamma_{5} e^{-\kappa \sigma} \otimes M_{12} \otimes k \\
\gamma_{5} e^{-\kappa \sigma} \otimes M_{12}^{*} \otimes k^{*} & \gamma^{a} e_{a}^{\mu}\left(\partial_{\mu}+\ldots\right) \otimes 1_{3}
\end{array}\right),
$$

where $M_{12}=\mu\left(\begin{array}{l}0 \\ 1\end{array}\right), k$ is a $3 \times 3$ family mixing matrix, $\kappa^{-1}$ is the Planck scale, and the dots correspond to the spin-connection and derivatives of the $\sigma$ field. It was shown in [3] that the gravitational action associated with this geometry yields the Einstein-Hilbert action of the metric $g_{\mu \nu}=e_{\mu}^{a} e_{\nu}^{b} \eta_{a b}$, as well as a kinetic action for the scalar field $\sigma$. Comparing the Dirac operator (1) with the one used in constructing the standard model [2] we find that we must identify $H_{0}=\left\langle\mu e^{-\kappa \sigma}\right\rangle$, where $H_{0}$ is the vacuum expectation value (vev) of the Higgs field. In the gravitational part of the action, the field $\sigma$ appears only through derivatives and, in the Yang-Mills-Higgs action, it appears as a scale factor, in powers of $e^{-\kappa \sigma}$. There is no pure potential term for $\sigma$, and its vev is undetermined at the classical level. We shall show how $e^{-\kappa \sigma}$ may acquire a vev as a consequence of radiative corrections, and how this imposes severe constraints on the top quark mass and the Higgs mass.

The non-commutative construction usually produces models with a restricted set of parameters [2-4]. This is, however, not the case for the standard model where the same number of parameters is obtained as in the usual approach. To determine explicitly the parameters of the theory and the $\sigma$ couplings, we indicate briefly, and in a simplified way, the steps of [2] leading to the classical action. The leptons are 
arranged in three copies of the multiplet $L=\left(\begin{array}{c}\nu_{L} \\ e_{L}^{-} \\ e_{R}^{-}\end{array}\right)$with a Dirac operator $D_{l}$ of a form given by equation (1). To incorporate the quarks correctly, a bimodule with algebras $\mathcal{A}$ and $\mathcal{B}$ is introduced [2]. When acting on the leptonic Hilbert space, the elements $a^{i}, b^{i}, \cdots$ of the algebra $\mathcal{A}$ describing the non-commutative space have a representation $: a \rightarrow \operatorname{diag}\left(a_{1}, a_{2}\right)$, where $a_{1}$ is a $2 \times 2$ quaternionic matrix of continuous functions and $a_{2}$ is a continuous function. A one-form in $\Omega^{1}(\mathcal{A})$ is given by $\rho=$ $\sum_{i} a^{i} d b^{i}$, and the involutive map $\pi_{l}$ is defined by

$$
\pi_{l}(\rho)=\sum_{i} a^{i}\left[D_{l}, b^{i}\right]
$$

and this is easily evaluated to be

$$
\pi_{l}(\rho)=\left(\begin{array}{cc}
A_{1} & \gamma_{5} k e^{-\kappa \sigma} H \\
\gamma_{5} k e^{-\kappa \sigma} H^{*} & A_{2}
\end{array}\right)
$$

where $A_{1}$ and $A_{2}$ are the $\mathrm{U}(2)$ and $\mathrm{U}(1)$ gauge fields. The algebra $\mathcal{B}$ is taken to be $M_{1}(C) \oplus M_{3}(C)$ commuting with the action of $\mathcal{A}$, and the mass matrices in the Dirac operator taken to be zero when acting on the elements of $\mathcal{B}$. Then the one-form $\eta$ in $\Omega^{1}(\mathcal{B})$ has the simple form $\pi_{l}(\eta)=B_{1} \operatorname{diag}\left(1_{2}, 1\right)$. The leptonic action is

$$
<L,(D+\rho+\eta) L>=\int d^{4} x \bar{L}\left(D_{l}+\pi_{l}(\rho)+\pi_{l}(\eta)\right) L
$$

The leptonic part of the electroweak bosonic action is

$$
I_{l}=\operatorname{Tr}_{w}\left(C_{l}\left(\theta_{\rho}+\theta_{\eta}\right)^{2} D_{l}^{-4}+\right)
$$

where $T r_{w}$ is the Dixmier trace [2], $C_{l}$ is a constant element of $\mathcal{A}$, and $\theta_{\rho}=d \rho+\rho^{2}$ is the curvature. Similarly, the quarks are arranged in three copies of the multiplet $Q=$ $\left(\begin{array}{l}u_{L} \\ d_{L} \\ d_{R} \\ u_{R}\end{array}\right)$. The elements of the algebra $\mathcal{A}$ have the representation $a \rightarrow \operatorname{diag}\left(a_{1}, a_{2}, \overline{a_{2}}\right)$ where $a_{1}$ is a $2 \times 2$ quaternionic matrix of continuous functions and $a_{2}$ is a complex valued continuous function. The Dirac operator $D_{q}$ associated with this representation is

$$
D_{q}=\left(\begin{array}{ccc}
\gamma^{a} e_{a}^{\mu}\left(\partial_{\mu}+\ldots\right) \otimes 1_{2} \otimes 1_{3} & \gamma_{5} e^{-\kappa \sigma} \otimes M_{12} \otimes k^{\prime} & \gamma_{5} e^{-\kappa \sigma} \otimes \tilde{M}_{12} \otimes k^{\prime \prime} \\
\gamma_{5} e^{-\kappa \sigma} \otimes M_{12}^{*} \otimes k^{\prime *} & \gamma^{a} e_{a}^{\mu}\left(\partial_{\mu}+\ldots\right) \otimes 1_{3} & 0 \\
\gamma_{5} e^{-\kappa \sigma} \otimes \tilde{M}_{12}{ }^{*} \otimes k^{\prime \prime} * & 0 & \gamma^{a} e_{a}^{\mu}\left(\partial_{\mu}+\ldots\right) \otimes 1_{3}
\end{array}\right)
$$


where $k^{\prime}$ and $k^{\prime \prime}$ are $3 \times 3$ family mixing matrices, and $\tilde{M}_{12}=\mu\left(\begin{array}{l}1 \\ 0\end{array}\right)$. Then the one-form in $\Omega^{1}(\mathcal{A})$ has the representation

$$
\pi_{q}(\rho)=\left(\begin{array}{ccc}
A_{1} \otimes 1_{3} & \gamma_{5} H \otimes k^{\prime} & \gamma_{5} \tilde{H} \otimes k^{\prime \prime} \\
\gamma_{5} H^{*} \otimes k^{*} & A_{2} \otimes 1_{3} & \frac{0}{A_{2}} \\
\gamma_{5} \tilde{H}^{*} \otimes k^{\prime \prime} & 0 &
\end{array}\right),
$$

where $\tilde{H}_{a}=\epsilon_{a b} H^{b}$. On the algebra $\mathcal{B}$ the Dirac operator has zero mass matrices, and the one form $\eta$ in $\Omega^{1}(\mathcal{B})$ has the representation $\pi_{q}(\eta)=B_{2} \operatorname{diag}\left(1_{2}, 1,1\right)$. Imposing the unimodularity condition on the algebras $\mathcal{A}$ and $\mathcal{B}$ relates the $\mathrm{U}(1)$ factors in both algebras: $\operatorname{tr}\left(A_{1}\right)=0, A_{2}=B_{1}=-\operatorname{tr} B_{2}=\frac{i}{2} g_{1} B$. We can then write

$$
\begin{aligned}
& A_{1}=-\frac{i}{2} g_{2} A^{a} \sigma_{a} \\
& B_{2}=-\frac{i}{6} g_{1} B-\frac{i}{2} g_{3} V^{i} \lambda_{i}
\end{aligned}
$$

where $g_{1}, g_{2}, g_{3}$ are the $\mathrm{U}(1), \mathrm{SU}(2)$, and SU(3) coupling constants, and $\sigma^{a}$ and $\lambda^{i}$ are the Pauli and Gell-Mann matrices respectively. The quark part of the electroweak bosonic action is

$$
I_{q}=\operatorname{Tr}\left(C_{q}\left(\theta_{\rho}^{2}+\theta_{\eta}^{2}\right) D_{q}^{-4}\right)
$$

By writing $C_{l}=\operatorname{diag}\left(c_{1}, c_{1}, c_{2}\right)$ and $C_{q}=\operatorname{diag}\left(c_{3}, c_{3}, c_{4}, c_{4}\right)$, the bosonic action depends on the constants $c_{1}, c_{2}, c_{3}, c_{4}, g_{1}, g_{2}, g_{3}$ as well as on the Yukawa couplings and on $e^{-\kappa \sigma}$. Normalizing the kinetic energies of the $S U(3), S U(2)$ and $U(1)$ gauge fields fixes three of the constants $c_{1}, \ldots, c_{4}$ in terms of $g_{1}, g_{2}, g_{3}$. In the special case when $c_{1}=c_{2}=c_{3}=c_{4}$, one gets a constraint on the gauge coupling constants as well as fixed values for the Higgs mass and top quark mass. These relations cannot be maintained after quantization, as can be seen from the renormalization group equations for the coupling constants and the masses [5]. We shall not assume any such relations among the $c^{\prime} s$. The Higgs sector is then parametrized in terms of two parameters $\lambda$ and $m$ which are functions of of the $c^{\prime} s, k, k^{\prime}, k^{\prime \prime}$ and $\left\langle H_{0}\right\rangle$. The bosonic part of the standard model is

$$
\begin{aligned}
L_{b}=- & \frac{1}{4}\left(F_{\mu \nu}^{3} F^{\mu \nu 3}+F_{\mu \nu}^{2} F^{\mu \nu 2}+F_{\mu \nu}^{1} F^{\mu \nu 1}\right) \\
& +D_{\mu}\left(H+M_{12}\right)^{*} D_{\nu}\left(H+M_{12}\right) g^{\mu \nu} e^{-2 \kappa \sigma} . \\
& -\frac{\lambda}{24}|| H+\left.M_{12}\right|^{2}-\left.\left|M_{12}\right|^{2}\right|^{2} e^{-4 \kappa \sigma}
\end{aligned}
$$

The cosmological constant comes out to be zero, naturally, at the classical level. The $\sigma$ dependence in (9) is a consequence of the "Weyl invariance" of the actions (4) 
and (8) under the rescaling of the Dirac operator $D \rightarrow e^{-w} D$, as this implies $g_{\mu \nu} \rightarrow$ $e^{2 w} g_{\mu \nu}$ and $\kappa \sigma \rightarrow \kappa \sigma+w$. This can be easily seen from the scalings: $\pi(\rho) \rightarrow e^{-w} \pi(\rho)$ and $\pi(\theta) \rightarrow e^{-2 w} \pi(\theta)$. By redefining $H+M_{12} \rightarrow e^{\kappa \sigma} H$, the $H$ dependent terms in (9) become

$$
D_{\mu} H^{*} D^{\mu} H+\kappa \partial_{\mu}\left(H^{*} H\right) \partial^{\mu} \sigma+\kappa^{2} H^{*} H \partial_{\mu} \partial^{\mu} \sigma-\frac{\lambda}{24}\left|\left(H^{*} H\right)^{2}-\mu^{2} e^{-2 \kappa \sigma}\right|^{2} .
$$

The potential in (10) could be rewritten in the familiar form

$$
V_{0}=\frac{\lambda}{24}\left(H^{*} H\right)^{2}-\frac{1}{2} m^{2}\left(H^{*} H\right)+\frac{3}{2 \lambda} m^{4}
$$

where we have set $m^{2}=\frac{\lambda \mu^{2}}{6} e^{-2 \kappa \sigma}$, so that $m$ is now a field and not just a parameter. A similar potential has also been considered in [6] with a different motivation. The potential $V_{0}$ is of the same form as that of the standard model. At the electroweak scale, which is much smaller than the gravitational Planck scale, the Lagrangian we consider is renormalizable. The cosmological constant in the standard model can be tuned to zero but in the non-commutative construction it automatically comes out to be zero at the tree level. We therefore assume that, after renormalization, the bosonic action takes the same form as $I_{l}+I_{q}$. We warn the reader that, although this assumption appears to be reasonable, we cannot prove that the most general form of a non-commutative Yang-Mills action is preserved at the quantum level, in the absence of some understanding of its symmetries. We shall proceed in our analysis on the basis of this assumption.

Let $\phi$ be the component of the Higgs field that develop a vev. We are then mainly interested in the potential

$$
V_{0}=\frac{\lambda}{24} \phi^{4}-\frac{1}{2} m^{2} \phi^{2}+\frac{3}{2 \lambda} m^{4}
$$

Minimizing with respect to $\phi$ and $m$ yields the equations

$$
\begin{aligned}
& 0=\frac{\lambda}{6} \phi^{3}-m^{2} \phi, \\
& 0=-m \phi^{2}+\frac{6}{\lambda} m^{3} .
\end{aligned}
$$

Both equations, (13) and (14), have the same asymmetric phase

$$
\phi^{2}=\frac{6}{\lambda} m^{2}
$$


and the weak scale, $e^{-\kappa \sigma}$, is undetermined at the classical level. The quantum corrections to the potential are given, in the one-loop approximation, by the effective Coleman-Weinberg [7] potential of the standard model [8]:

$$
\begin{gathered}
V_{1}=\frac{1}{16 \pi^{2}}\left(\frac{1}{4} H^{2}\left(\ln \frac{H}{M^{2}}-\frac{3}{2}\right)+\frac{3}{4} G^{2}\left(\ln \frac{G}{M^{2}}-\frac{3}{2}\right)+\frac{3}{2} W^{2}\left(\ln \frac{W}{M^{2}}-\frac{5}{6}\right)\right. \\
\left.+\frac{3}{4} Z^{2}\left(\ln \frac{Z}{M^{2}}-\frac{5}{6}\right)-3 T^{2}\left(\ln \frac{T}{M^{2}}-\frac{3}{2}\right)\right)
\end{gathered},
$$

where

$$
\begin{aligned}
& H=-m^{2}+\frac{1}{2} \lambda \phi^{2}, \quad G=-m^{2}+\frac{1}{6} \lambda \phi^{2}, \\
& W=\frac{1}{4} g_{2}^{2} \phi^{2}, \quad Z=\frac{1}{4}\left(g_{2}^{2}+g_{1}^{2}\right) \phi^{2}, \quad T=\frac{1}{2} h^{2} \phi^{2},
\end{aligned}
$$

and $M$ is the renormalization scale. At the classical minimmum $\phi^{2}=\frac{6}{\lambda} m^{2}, H, W, Z$ and $T$ are respectively, the squares of the masses of the Higgs, $W^{ \pm}, \mathrm{Z}$ and t particles. The potential $V_{1}$ is independent of $M$ because the coupling constans $g_{1}, g_{2}$, $h$, and $\lambda$ depend on $M$ through the renormalization group equations in such a way that $\frac{\partial V_{1}}{\partial M}=0$.

Minimizing the total potential $V_{0}+V_{1}$ with respect to the fields $\phi$ and $m$ gives respectively

$$
\begin{aligned}
& 0=\phi(G+ \frac{1}{32 \pi^{2}}\left(\lambda H\left(\ln \frac{H}{M^{2}}-1\right)+\lambda G\left(\ln \frac{G}{M^{2}}-1\right)-12 h^{2} T\left(\ln \frac{T}{M^{2}}-1\right)\right. \\
&\left.\left.+3 g_{2}^{2} W\left(\ln \frac{W}{M^{2}}-\frac{1}{3}\right)+\frac{3}{2}\left(g_{2}^{2}+g_{1}^{2}\right) Z\left(\ln \frac{Z}{M^{2}}-\frac{1}{3}\right)\right)\right) \\
& 0=-m\left(\frac{6}{\lambda} G+\frac{1}{16 \pi^{2}}\left(H\left(\ln \frac{H}{M^{2}}-1\right)+3 G\left(\ln \frac{G}{M^{2}}-1\right)\right) .\right)
\end{aligned}
$$

At the scale $M=m_{Z}$, the mass of the Z-particle, the coupling constants $g_{1}, g_{2}$ as well as the vev $\phi$ are known from experimental data, corrected with the help of the renormalization group equations [8]:

$$
g_{2}=0.650, \quad g_{1}=0.358, \quad \phi=246 \mathrm{Gev}
$$

and this implies that

$$
W=m_{W}^{2}=6392.002 G e v^{2}, \quad Z=m_{Z}^{2}=8330.996 G e v^{2}
$$

The only unknowns in the minimization equations are $\lambda, m$ and the square of the top quark mass $T=m_{t}^{2}$. (In reality, $T$ is the sum of the squares of all the quark masses, but this is dominated by the top quark mass). We shall use the top quark mass as 
a parameter and solve equations (18) and (19) for the full range of this parameter. We first express $\lambda$ and $m^{2}$ in terms of $H$ and $G$ :

$$
\lambda=\frac{3}{\phi^{2}}(H-G), \quad m^{2}=\frac{1}{2}(H-3 G),
$$

and $h$ in terms of $T: h^{2}=\frac{2 T}{\phi^{2}}$. After rescaling

$$
G=\bar{G} M^{2}, \quad H=\bar{H} M^{2}, \quad T=\bar{T} M^{2}
$$

the asymmetric solution of equations (18) and (19) is given by the solution of the following two equations:

$$
\begin{aligned}
0=\bar{G} & +\frac{M^{2}}{32 \pi^{2} \phi^{2}}(\bar{H}-\bar{G})(\bar{H}(\ln \bar{H}-1)+3 \bar{G}(\ln \bar{G}-1)) \\
0=\bar{G} & +\frac{3 M^{2}}{32 \pi^{2} \phi^{2}}(\bar{H}-\bar{G})(\bar{H}(\ln \bar{H}-1)+\bar{G}(\ln \bar{G}-1))-\frac{g_{2}^{2}+g_{1}^{2}}{64 \pi^{2}} \\
& +\frac{3 g_{2}^{4} \phi^{2}}{128 \pi^{2} M^{2}}\left(\ln \frac{g_{2}^{2} \phi^{2}}{4 M^{2}}-\frac{1}{3}\right)-\frac{3 M^{2}}{4 \pi^{2} \phi^{2}} \bar{T}^{2}(\ln \bar{T}-1) .
\end{aligned}
$$

These equations, being complicated functions of $\bar{H}$ and $\bar{G}$, could only be solved numerically, for various values of $\bar{T}$. The numerical solutions were easily obtained using Mathematica. Before presenting the solutions, we note that for a given value of $\bar{H}, \bar{G}$, and $\bar{T}$, the Higgs mass can be determined from the formula $m_{H}^{2}=\frac{\partial^{2} V}{\partial \phi^{2}}$ which gives

$$
\begin{gathered}
m_{H}^{2}=M^{2}\left((\bar{H}-\bar{G})+\frac{9 M^{2}}{16 \pi^{2} \phi^{2}}(\bar{H}-\bar{G})^{2}\left(\ln \bar{H}+\frac{1}{3} \ln \bar{G}\right)\right. \\
\left.+\frac{3 g_{2}^{4} \phi^{2}}{64 \pi^{2} M^{2}} \ln \frac{g_{2}^{2} \phi^{2}}{4 M^{2}}-\frac{3 M^{2}}{2 \pi^{2} \phi^{2}} \bar{T}^{2} \ln \bar{T}\right)
\end{gathered}
$$

We now quote the results: There are only two classes of solutions, for $\bar{G} \ll \bar{H}$ and for $\bar{H} \ll \bar{G}$. In the first case we find that there are only two narrow bands for the top quark mass where solutions exist. The first band is

$$
0.365 \leq \bar{T} \leq 0.455, \quad \bar{G} \ll \bar{H}
$$

corresponding to a top quark mass $54.90 \leq m_{t} \leq 61.35 \mathrm{Gev}$ which is already ruled out experimentally. The second band is very narrow:

$$
2.57 \leq \bar{T} \leq 2.61, \quad \bar{G} \ll \bar{H},
$$

corresponding to the top quark mass

$$
146.23 \leq m_{t} \leq 147.37 \text { Gev }
$$


and a Higgs mass $117.26 \leq m_{H} \leq 142.61$ Gev. Clearly this band of values for the top quark mass lies within the present experimental average of [9]

$$
m_{t}=149+\left(\begin{array}{c}
+21 \\
-47
\end{array}\right) \text { Gev. }
$$

These solutions are presented in Tables 1 and 2. The second class of solutions occurs when

$$
1.30 \leq \bar{T} \leq 2.61, \quad \bar{H} \ll \bar{G},
$$

corresponding to the top quark mass $104.07 \leq m_{t} \leq 147.48 \mathrm{Gev}$, and a Higgs mass $1208 \geq m_{H} \geq 1197$ Gev. These solutions are given in Table 3. However, since $\bar{H} \ll \bar{G}$, and since the coupling constant $\lambda=O(-100)$, the potential, in this domain, becomes unbounded from below, signaling the break down of the perturbative region. Requiring stability of the electroweak potential excludes this solution. Therefore the only accptable solution is (25) which is remarkably constrained, considering the wide range of possibilities that one might have apriori.

To have a better understanding of the solutions obtained, we rewrite eqs (24), (25) using the numerical values (20), (21):

$$
\begin{gathered}
(\bar{H}-\bar{G}) \bar{H}(\ln \bar{H}-1)=12 \bar{T}^{2}(\ln \bar{T}-1)+3.113136, \\
\bar{G}+4.3589^{-4}(\bar{H}-\bar{G})(\bar{H}(\ln \bar{H}-1)+3 \bar{G}(\ln \bar{G}-1))=0 .
\end{gathered}
$$

The right-hand side of eq (32) is zero at $\bar{T}=0.357644$ and at $\bar{T}=2.61727$, becomes negative for the values of $\bar{T}$ in between and positive otherwise. From eq (33) we see that if the right-hand side of eq (32) is negative, then $\bar{G} \equiv O\left(10^{-4}\right)$ and $\bar{G} \ll \bar{H}$. Ignoring $\bar{G}$ in eq (32), the left-hand side of this equation becomes negative for $0<$ $\bar{H}<e$, with a minimum value of $-\frac{e}{2}$ at $\bar{H}=\sqrt{e}$. The right-hand side of eq (32) is larger than $-\frac{e}{2}$, for $2.569 \leq \bar{T} \leq 2.617$ and $0.357 \leq \bar{T} \leq 0.457$. The numerical solutions, taking $\bar{G}$ into account, are shown in Tables 1 and 2 . When the right-hand side of eq (32) is positive then $\bar{H}>\bar{G}$ and $\bar{H}>e$, or $\bar{H}<\bar{G}$ and $\bar{H}<e$. In the first case, since $\bar{G}$ must be positive for the potential not to become complex, one finds that there are no solutions. In the second case solutions would exist if $\bar{H} \ll \bar{G}$, giving rise to large negative coupling constant $\lambda$, and therefore, physically unacceptable. These solutions are shown in Table 3.

We note that the field $\sigma$ becomes massive with the square of the mass given by: $m_{\sigma}^{2}=\frac{\partial^{2} V}{\partial \sigma^{2}}$ and this is equal to

$$
m_{\sigma}^{2}=\kappa^{2} m^{2}\left(2 \phi^{2} \frac{\bar{H}-4 \bar{G}}{\bar{H}-\bar{G}}+\frac{M^{2}}{16 \pi^{2}}(\bar{H}(1-\ln \bar{H})+3 \bar{G}(1-\ln \bar{G}))\right) .
$$


For the physically acceptable solutions we have $\bar{H}=O(1), \bar{G}=O\left(10^{-4}\right)$ and $m^{2}=$ $O\left(M^{2}\right)$. Then we find from eq (29) that

$$
m_{\sigma}^{2}=O\left(\kappa^{2} M^{4}\right),
$$

so that $m_{\sigma}=O\left(10^{-15}\right) \mathrm{Gev}$, which is unobservable.

To summarize, we have shown that the only acceptable solutions for the minimization of the total potential exist in the narrow band (25) of the top quark mass and the Higgs mass. Of course, these predictions have, at best, heuristic value, since the problem of fixing the form of the cosmological constant at the one-loop level by imposing natural geometrical constraints is not understood. However, they do suggest that gravitational effects may play a role in understanding masses of fermions and Higgses and that methods of non-commutative geometry may be useful in making progress on these problems.

\section{Acknowledgments}

We would like to thank D. Wyler for very useful discussions, and Z. Trocsanyi for help in computer programming. A.H.C would like to thank D. Kastler for illuminating discussions, the Université Aix Marseille II and the Centre de Physique Théorique Marseille, where part of this work was done, for hospitality. 


\section{References}

[1] A. Connes, Publ. Math. IHES 6244 (1983);

A. Connes, in the interface of mathematics and particle physics, Clarendon press, Oxford 1990, Eds D. Quillen, G. Segal and S. Tsou.

[2] A. Connes and J. Lott,Nucl.Phys.B Proc.Supp. 18B 29 (1990), North-Holland, Amsterdam; Proceedings of 1991 Summer Cargese conference p.53 editors J. Fröhlich et al (1992),Plenum Pub.;

For a detailed account see: D. Kastler, Marseille preprints CPT-91/P.2610, CPT-91/P.2611 and forthcoming book.

[3] A.H. Chamseddine, G. Felder and J. Fröhlich, Zurich preprint ZU-TH-30 1992, to appear in Comm.Math.Phys.

[4] R. Coquereaux, G. Esposito-Farése, G. Vaillant, Nucl. Phys.B353 689 (1991);

M. Dubois-Violette, R. Kerner, J. Madore, J. Math. Phys.31 (1990) 316;

R. Coquereaux, G. Esposito-Farése and F. Scheck, Int.J.Mod.Phys. A7 (1992) 6555 ;

B. Balakrishna, F. Gürsey and K. C. Wali, Phys. Lett. 254B (1991) 430;

A.H. Chamseddine, G. Felder and J. Fröhlich, Nucl.Phys. B395 (1993) 672.

[5] E. Alvarez, J.M. Garcia-Bondia and C.P. Martin, Madrid preprint, May 1993.

[6] W. Buchmüller and D. Wyler, Phys.Lett B249 (1990) 281.

[7] E. Weinberg and S. Coleman, Phys.RevD7 (1973)1888.

[8] M. Sher, Phys.Rep179(1989) 274 and references therein;

C. Ford, D.R.T. Jones, Y.W. Stephenson and M.B. Einhorn, Liverpool preprint LTH 288.

[9] U. Amaldi, to appear in Proceedings of Salamfest ,Trieste, Italy, (March 1993), editor S. Randjbar-Daemi, World-Scientific, Singapore. 


\begin{tabular}{|c|c|c|c|c|}
\hline \multicolumn{5}{|c|}{ Table $1 \quad(\bar{G} \ll \bar{H})$} \\
\hline $\bar{T}$ & $\bar{G}$ & $\bar{H}$ & $m_{t}(\mathrm{Gev})$ & $m_{H}(\mathrm{Gev})$ \\
\hline .360 & .0000141 & 2.707 & 54.518 & 148.001 \\
\hline .370 & .0000738 & 2.656 & 55.276 & 147.079 \\
\hline .380 & .0001337 & 2.602 & 56.025 & 145.738 \\
\hline .390 & .0001934 & 2.543 & 56.763 & 144.194 \\
\hline .400 & .0002544 & 2.479 & 57.493 & 142.446 \\
\hline .410 & .0003152 & 2.408 & 58.213 & 140.459 \\
\hline .420 & .0003763 & 2.328 & 58.924 & 138.159 \\
\hline .430 & .0004376 & 2.235 & 59.627 & 135.413 \\
\hline .440 & .0004989 & 2.120 & 60.321 & 131.935 \\
\hline .450 & .0005602 & 1.958 & 61.008 & 126.869 \\
\hline .455 & .0005905 & 1.823 & 61.348 & 122.434 \\
\hline
\end{tabular}

\begin{tabular}{|c|c|c|c|c|}
\hline \multicolumn{5}{|c|}{ Table $2(\bar{G} \ll \bar{H})$} \\
\hline $\bar{T}$ & $\bar{G}$ & $\bar{H}$ & $m_{t}(\mathrm{Gev})$ & $m_{H}(\mathrm{Gev})$ \\
\hline 2.570 & .0005928 & 1.807 & 146.231 & 117.257 \\
\hline 2.575 & .0005332 & 2.039 & 146.374 & 125.012 \\
\hline 2.580 & .0004725 & 2.173 & 146.516 & 129.267 \\
\hline 2.585 & .0004110 & 2.277 & 146.658 & 132.461 \\
\hline 2.590 & .0003489 & 2.365 & 146.800 & 135.083 \\
\hline 2.595 & .0002862 & 2.443 & 146.942 & 137.332 \\
\hline 2.600 & .0002230 & 2.513 & 147.084 & 139.307 \\
\hline 2.605 & .0001592 & 2.578 & 147.225 & 141.062 \\
\hline 2.610 & .0000948 & 2.638 & 147.367 & 142.612 \\
\hline
\end{tabular}

\begin{tabular}{|c|c|c|c|c|}
\hline \multicolumn{5}{|c|}{ Table $3 \quad(\bar{G}>\bar{H}$} \\
\hline $\bar{T}$ & $\bar{G}$ & $\bar{H}$ & $m_{t}(\mathrm{Gev})$ & $m_{H}(\mathrm{Gev})$ \\
\hline 1.30 & 184.169 & 2.783 & 104.069 & 1270.816 \\
\hline 1.50 & 184.173 & 2.789 & 111.788 & 1208.710 \\
\hline 1.70 & 184.174 & 2.790 & 119.007 & 1208.856 \\
\hline 1.90 & 184.171 & 2.786 & 125.813 & 1208.153 \\
\hline 2.10 & 184.164 & 2.776 & 132.269 & 1206.499 \\
\hline 2.30 & 184.151 & 2.759 & 138.424 & 1203.787 \\
\hline 2.50 & 184.134 & 2.736 & 144.317 & 1199.892 \\
\hline 2.61 & 184.122 & 2.719 & 147.458 & 1197.194 \\
\hline
\end{tabular}

